has maintained organized co-operative industrial research. Dr. J. G. King, formerly of the Fuel Research Station, has been appointed director, and Dr. F. J. Dent, who has been responsible for a large part of the research activity of the Joint Research Committee of the Gas Research Board and the University of Leeds, has been appointed joint assistant director.

Of the items mentioned, gas technologists will note with interest the progress in the work on the complete gasification of coal. This shows that several lines of work are being followed-direct hydrogenation under pressure, gasification in oxygen and steam under pressure, and catalytic synthesis of hydrocarbons from carbon monoxide and hydrogen. The laboratory work on these themes is in course of transference to large-scale working in plant erected in a provincial gas works. Research on gas purification holds promise of reducing the sulphur content of purified coal gas to one tenth of the figures currently obtained in public supply in Great Britain. Drying by infra-red radiation emitted by gas-heated sources is being studied and apparently offers certain advantages. Methane, a gas of high calorific value, is a principal constituent of coal gas, from which it can be separated by liquefaction to give a portable liquid fuel which in future may te of great service. Gas engineers have always taken great interest in refractory materials, and the present report again reveals this interest. The report may be said to show that the research association under its new style shows already a wide and widening range of activities.

\section{Terms Used in Telecommunication}

THE modern rapid growth of the applications of radio and telecommunications technique makes it desirable, even in war-time, to keep as up to date as possible a collection of definitions of the various terms and phrases used by workers and stưdents in this field. To give effect to this point of view, the British Standards Institution has just issued a revised and enlarged edition of B.S. 204 entitled "Glossary of Terms used in Telecommunication" (obtainable from the British Standards Institution, 28 Victoria Street, London, S.W.1, 2s.). This publication has been prepared in collaboration with the General Post Ofice and other organizations concerned with communications technique and practice. It comprises revised sections of earlier glossaries dealing with telegraphy, telephony, radiocommunication, television and radio direction-finding, together with a new section on fire alarms. An appendix collects together the various symbols used for the quantities defined in the glossary. While it is doubtful if all workers in this field will agree with all the definitions, the revision, collection and rearrangement of the terms in this new publication will be found of considerable use as a reference manual by all those concerned with the preparation of technical documents and publications, as well as by the large number of other scientific and technical workers in this rapidly expanding field of telecommunications.

\section{Radio-Telegraph Signals}

A PAPER on high-speed recording of radio-telegraph signals was read recently in London before the Institution of Electrical Engineers by Messrs. R. B. Armstrong and J. A. Smale, in which the authors first describe the systems in most general use, and then give a brief definition of modulation requirements for telegraph services. The various sources of distortion encountered are fading, noise and interference from other stations, but chiefly phase distortion due to propagation over more than one route between transmitter and receiver. The on-and-off character of Morse signalling enhances the difficulties which come from most sources of distortion. The paper then describes the general characteristics required in radio receivers designed for the purposes under discussion, including a description of two types of receiver in current use. This is followed by a consideration of special requirements of the recording units into which the receivers work, with a description of a typical unit. The special measures provided to offset the three types of distortion previously mentioned are also dealt with.

Diversity reception is discussed, with particular reference to the special problems of combining the automatic gain-control systems and the receiver outputs. Recording by undulator is chiefly considered in the paper as a whole; but the discussion on the effects of distortion, and the counter-measures taken, is even more applicable to machine-printing systems, since the latter have less margins of tolerance in operation. The paper concludes with an indication of the trend of development towards different methods of signalling, which may reduce difficulties of reception and recording in comparison with the old on-and-off methods of conveying intelligence.

\section{Lighting Reconstruction}

The Illuminating Engineering Society has just issued the first three of a series of Lighting Reconstruction Pamphlets which are planned to be of service to Govermment departments, local authorities, borough engineers, architects and others who are preparing now for the lighting problems which will confront Great Britain during the period of post-war reconstruction. The present pamphlets relate respectively to "Principles of Good Lighting", "The Lighting of Public Buildings" and "The Lighting of Schools", and they provide excellent summaries of the broad principles of what is needed to make lighting efficient according to the particular application. The pamphlets are obtainable from the Society at 32 Victoria Street, S.W.1, at the uniform price of $1 s$. each, $9 s$. per dozen, or $£ 3$ per 100 .

\section{Distilled Water}

THE development of the water-still has keen slow, the normal type of apparatus being an externally heated metal boiler and a condenser. These are very inefficient, due to the fact that the number of calories required to heat the incoming cold water to boiling point is small compared with the heat required to convert the water into steam, and many kilowatts are required for an output of 20 gallons per hour. There is also the disadvantage of fur deposition from hard water, which can be diminished by taking only a fraction of the rated output.

A new type of still, called the "Strip-Action Still", is announced by Messrs. Townson and Mercer Ltd., 390 Sydenham Road, Croydon, which uses raw steam such as is available in a factory, and is made in units with a capacity of one gallon per hour. The initial design was in heat-resisting glass. The steam passes through an outer jacket which is air-cooled, and deposits dirt and high-boiling liquids with part of the condensed steam. The clean steam then passes down a multi-surface spiral condenser and comes out at the bottom as distilled water. By avoiding much contact with air, the water is of appreciably better 